\title{
Tiny Crystals Located and Identified by Infrared Nanoscopy
}

\section{Clemens Liewald ${ }^{1 *}$ and Fritz Keilmann ${ }^{1,2}$}

${ }^{1}$ Ludwig-Maximilians-Universität and Center for NanoScience, 80539 München, Germany

${ }^{2} \mathrm{Neaspec} \mathrm{GmbH}, 82152$ Martinsried, Germany

*Clemens.Liewald@physik.uni-muenchen.de

\section{Introduction}

Infrared (IR) spectroscopic analysis of materials is based on molecular vibrations that label any chemical compound with a characteristic "fingerprint" spectrum in the $3-30 \mu \mathrm{m}$ wavelength region. No labeling of samples is needed for IR spectrometers (usually abbreviated FTIR for Fourier-transform infrared spectrometer) to readily acquire IR spectra of any material in any form, be it in gas, liquid, or solid state. Infrared spectra identify chemicals in a straightforward manner, and therefore most suppliers bundle their FTIR instruments with spectral databases that offer automatic chemical recognition and even quantitative information (chemometrics) on the relative abundance of individual constituents of mixtures and composite materials. No wonder IR spectrometers are helpful in all fields of science and engineering and are common workhorses in university and industrial laboratories.

The single worst shortcoming of IR chemical recognition has been its inability to perform submicrometric microscopy, owing to the diffraction limit of spatial resolution, at half the wavelength of light analyzed. In the world of sub- $\mu \mathrm{m}$ microscopy, IR was a "no go" in spite of the inherent rich information available. But there is no fundamental-physics reason why light could not be concentrated to a volume much smaller than a wavelength, even down to atomic size. Fortunately an 85-year-old idea on super-focusing was turned into reality two decades ago when antenna-based light concentration to a few nm size was demonstrated in theory and experiment [1]. In consequence light optical microscopy and IR near-field microscopy could be successfully developed in a single instrument [2], and a commercial optical/IR/THz nanoscope product was introduced (neaspec.com) that offers a routine spatial resolution of $20 \mathrm{~nm}$. Named NeaSNOM (for new scanning near-field optical microscope) it earned an Innovation Award from Microscopy Today in 2013. Here we highlight the real-world application of NeaSNOM, including recent searches for sub- $\mu \mathrm{m}$ crystals in a biological context $[3,4]$, and sub- $\mu \mathrm{m}$ coexisting structural phases within organic thin films [5].

\section{Materials and Methods}

Basics of IR nanoscopy. A standard cantilevered tip (metallized version), as used in any atomic force microscope (AFM), serves as the infrared-concentrating element: it is illuminated by a conventionally focused IR beam and develops a tiny "hot spot" of greatly enhanced intensity just under the tip (Figure 1a). When the tip is close to the sample, the photons in this spot get modified in their amplitude and phase in response to the complex refractive index of the sample at just this location. This information about the sample is carried with the IR radiation scattered in all directions. Back-scattered
IR light is collected for the convenience of the scatteringtype SNOM's (s-SNOM's) instrument operator. Proprietary electronic filtering selects the photons from just the hot spot by capitalizing on the effect of tapping at 10-50 $\mathrm{nm}$ amplitude, which turns the near-field interaction between tip and sample off and on at the cantilever's mechanical oscillation frequency of around $100 \mathrm{kHz}$ [2].

a

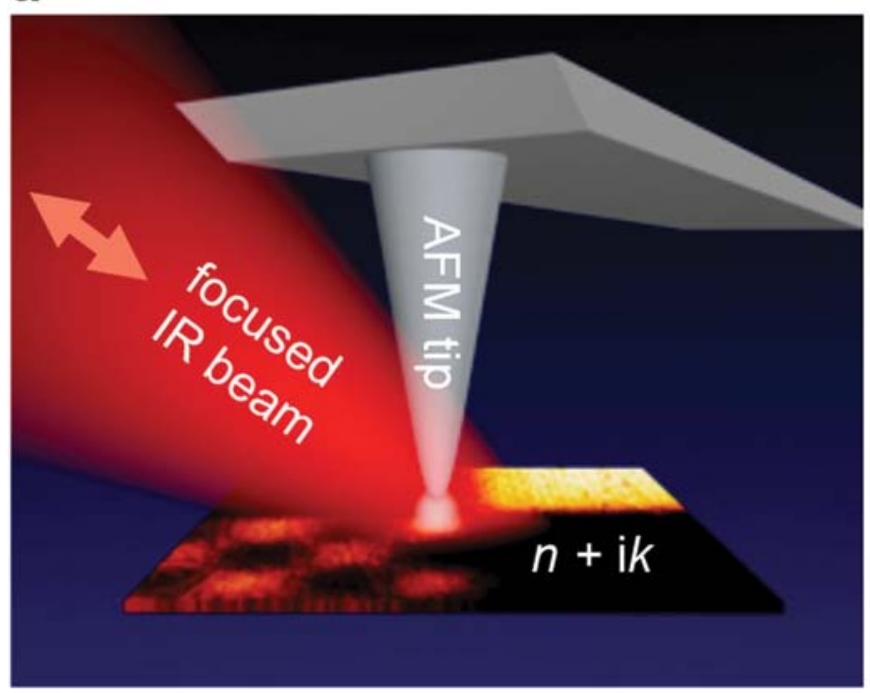

b

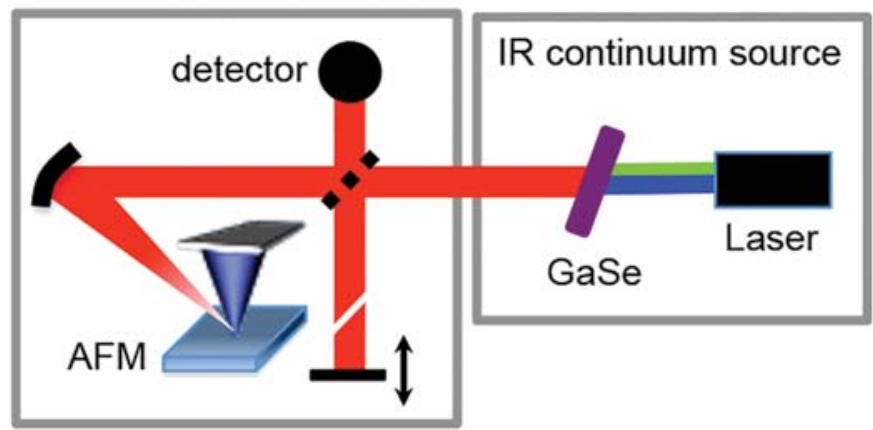

Figure 1: (a) Schematic light path in the IR near-field nanoscope, where an AFM tip operates in tapping mode to map the topography, while focused IR side-illumination creates a probing "hot spot," which is as small as the tip diameter, typically $20 \mathrm{~nm}$, independent of wavelength in the IR and THz regions. Back-scattered IR is detected and mapped simultaneously with the topography, producing IR contrast determined by the local refractive index $n+i k$. (b) Nano-FTIR mode of operating an IR s-SNOM. Illumination is by a broadband IR source consisting of a pulsed laser driving a crystalline GaSe frequency converter, while detection is via a built-in Michelson interferometer and subsequent Fourier-transformation. Complete IR amplitude and IR phase spectra are determined at each pixel of the scanned image. 


\section{Revolutionize Your Confocal Imaging ZEISS LSM $\mathbf{8 8 0}$ with Airyscan}
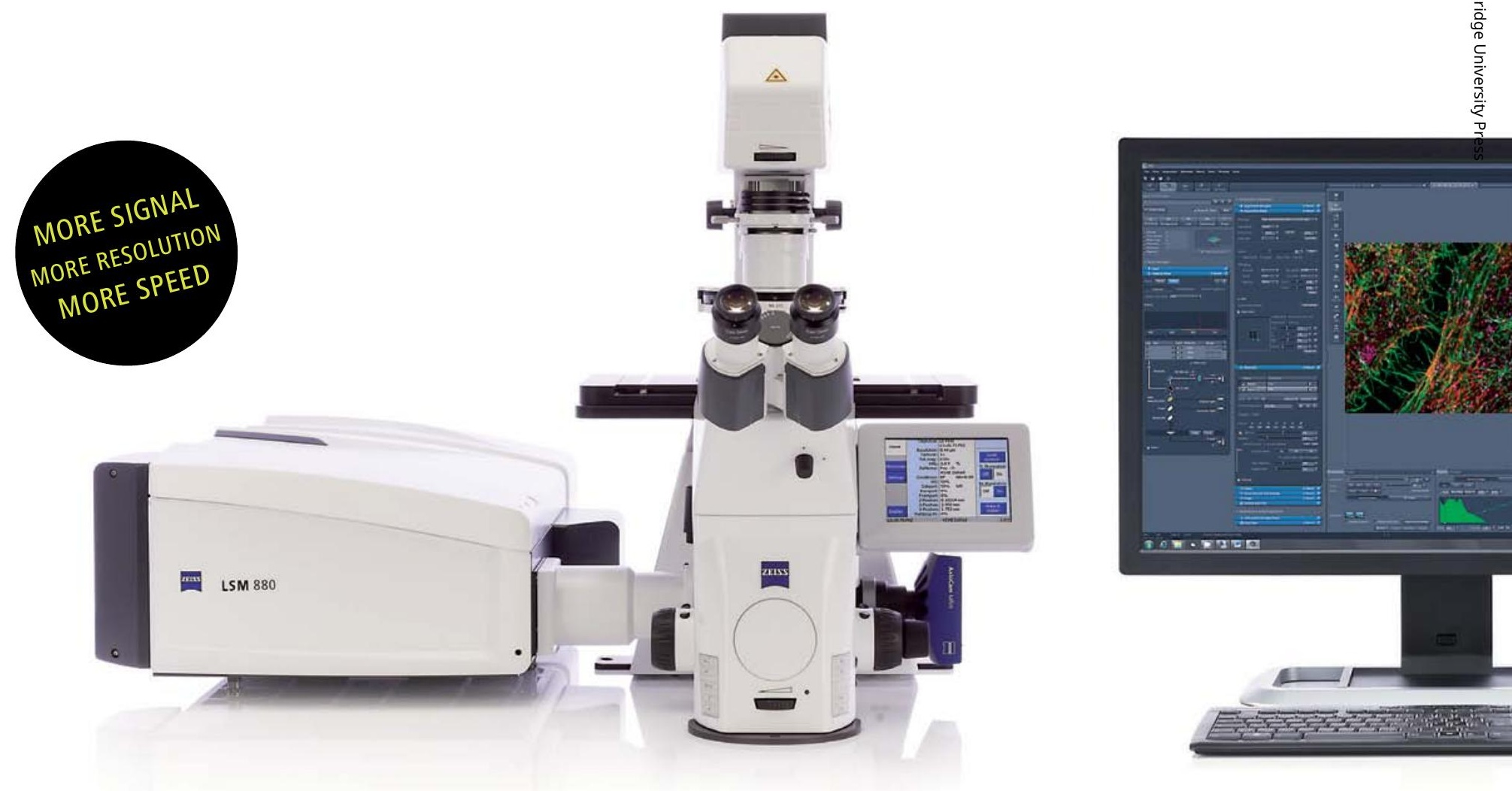

\section{Revolutionize Your Confocal Imaging}

Discover ZEISS LSM 880 with Airyscan - the new confocal laser scanning microscope that offers high sensitivity, improved resolution in $x, y$ and $z$, and high speed. All in one system. Find out more and book a hands-on demonstration in one of our ZEISS Microscopy Labs now.

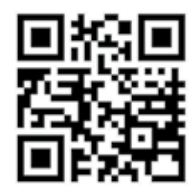

\section{ZEINXY}


Acquisition modes. The NeaSNOM nanoscope offers alternation between two modes of acquiring and displaying IR information, monochromatic IR images and hyperspectral maps. More precisely, the nanoscope can output an image at a fixed wavelength chosen to highlight a desired molecular component (or a series of repeated images at varied wavelengths to obtain rudimentary spectral information), but it can also be switched to deliver broad IR spectra, pixel-by-pixel, for local chemical recognition and identification even of unexpected material. The latter configuration, termed nano-FTIR (Figure 1b), includes all essential parts of an FTIR spectrometer with the nanoscope namely a broadband IR source and a Michelson interferometer (but unlike standard FTIR, the nano-FTIR uses an asymmetric configuration that can determine both amplitude and phase spectra) [6].

\section{Results}

Phosphate flakes on a biological specimen. One application potential of s-SNOM is for biological and medical purposes, by imaging microscopic biomineral structures [3]. Figure 2a shows images of a polished cross section of a mussel shell consisting of calcium carbonate microcrystals bound by organic material. The calcitic crystal structure in the outer part of the shell (top) abruptly transitions to the periodic arrangement of aragonite microcrystals in the inner part (bottom), leading to the well-known mother-of-pearl iridescent appearance of structural color. With monochromatic IR illumination selected at $980 \mathrm{~cm}^{-1}$ (corresponding to a wavelength of $10.2 \mu \mathrm{m}$ ), which is away from calcium carbonate vibration frequencies, the IR image shows no contrast between these types of microcrystals, but rather, a consistently higher signal than the organic material in between. Notice that the differing topographical height of the calcite surfaces does not change the observed IR signal, as the latter only depends on refractive index; there is no topography artifact or cross-talk into the IR image.

Surprisingly, numerous nano-scale features of enhanced scattering were discovered (yellow in the chosen color scale) and shown in Figure 2a. Close inspection of the topography verified that these are about $10 \mathrm{~nm}$ high objects. In order to learn about the chemical nature of these unexpected objects, the same location was investigated by the nano-FTIR mode of operation. Three-hundred IR spectra were collected along a line crossing one of the objects and extending $2.5 \mu \mathrm{m}$ over several calcite and arogonite microcrystals. The result (Figure $2 \mathrm{~b}$ ) shows the expected fingerprint resonance due to the well-known crystal vibrations of trigonal calcite and orthorhomic aragonite, at 873 and $855 \mathrm{~cm}^{-1}$, respectively. The resonance at about $1,018 \mathrm{~cm}^{-1}$ identifies the thin object as crystalline phosphate. Close inspection (Figure 2a) reveals that all such flakes observed appear to be $10-20 \mathrm{~nm}$ high and have a smoother surface than the carbonate crystals. Even though these features are found in the calcite but not the aragonite, they were tentatively assigned to be just preparation artifacts. In any case, the important conclusion remains that s-SNOM and nano-FTIR are capable of not only detecting but also identifying tiny crystals on a sample surface.

Human bone. Bone samples were nanoscopically inspected after standard poly (methyl methacrylate) (PMMA) embedding and polishing. Figure 3 shows an IR s-SNOM
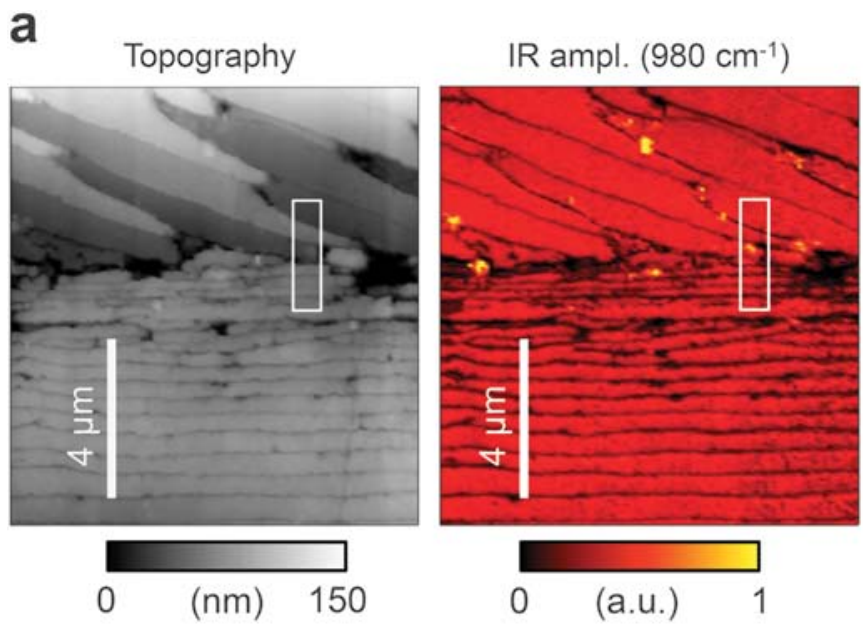

b

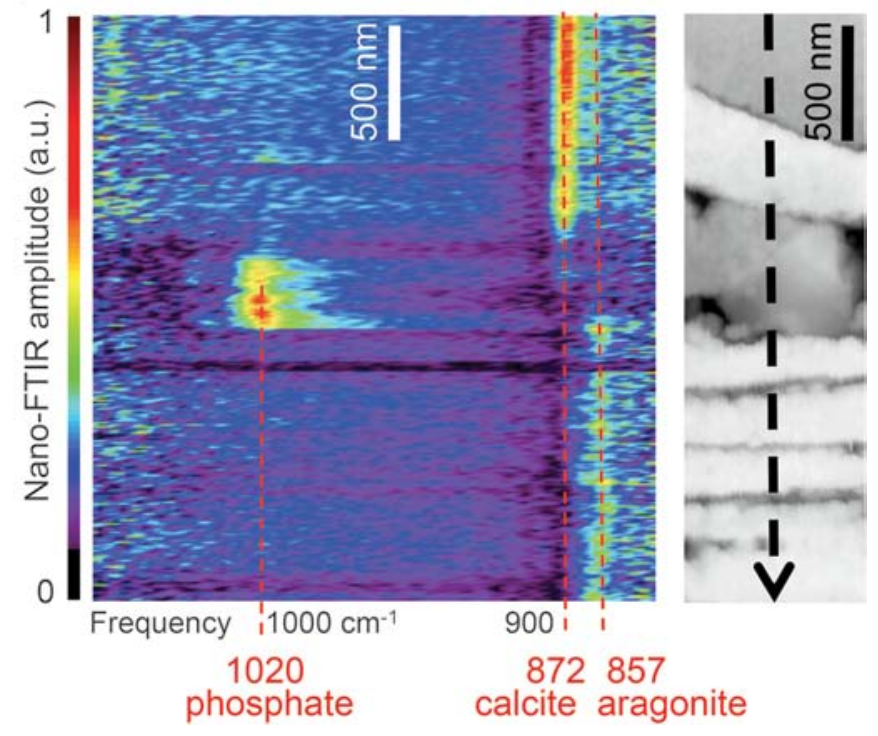

Figure 2: (a) IR nanoscope image $\left(10 \times 10 \mu \mathrm{m}^{2}\right)$ of a polished section through a mussel shell; topographical image (left) and back-scattering signal at $10.2 \mu \mathrm{m}$ wavelength (right). Both the IR and topography images show microcrystals at the same high resolution, known to be calcium carbonate of either the calcite or aragonite crystal structure. Both polymorphs exhibit the same IR signal because their vibration frequencies are far from the probing frequency, but the image also exhibits bright patches indicating a contaminating material (see text) [3]. (b) Nano-FTIR spectra along a line on the topography image depicted as rectangle. The line crosses a 10-nm thin object in the calcite-aragonite transition region of the mussel shell. Fingerprint vibration lines clearly distinguish both carbonate phases and identify the thin object as a $10 \mathrm{~nm}$ thin flake of crystalline phosphate [3].

image of a human femoral head at $9.47 \mu \mathrm{m}$ wavelength. This resonance is associated with the $\mathrm{PO}_{3}{ }^{-}$vibration of phosphate nanocrystals, the main mineral component of bone [4]. The marked void designates a canaliculus. It is surrounded by a $1 \mu \mathrm{m}$ wide region of increased IR signal, which indicates a higher mineral density. The observed nanostructures were suspected to be fibrils of collagen with attached phosphate nanocrystals. The fibril orientation appears to be perpendicular to the sample surface in the neighborhood of the canaliculus, but not so further out. Further images were taken of lacunae [4], and also of tubuli in human dentin, and the structures were verified by scanning electron microscopy (SEM) and BE-SEM. Because SEM can damage the sample 


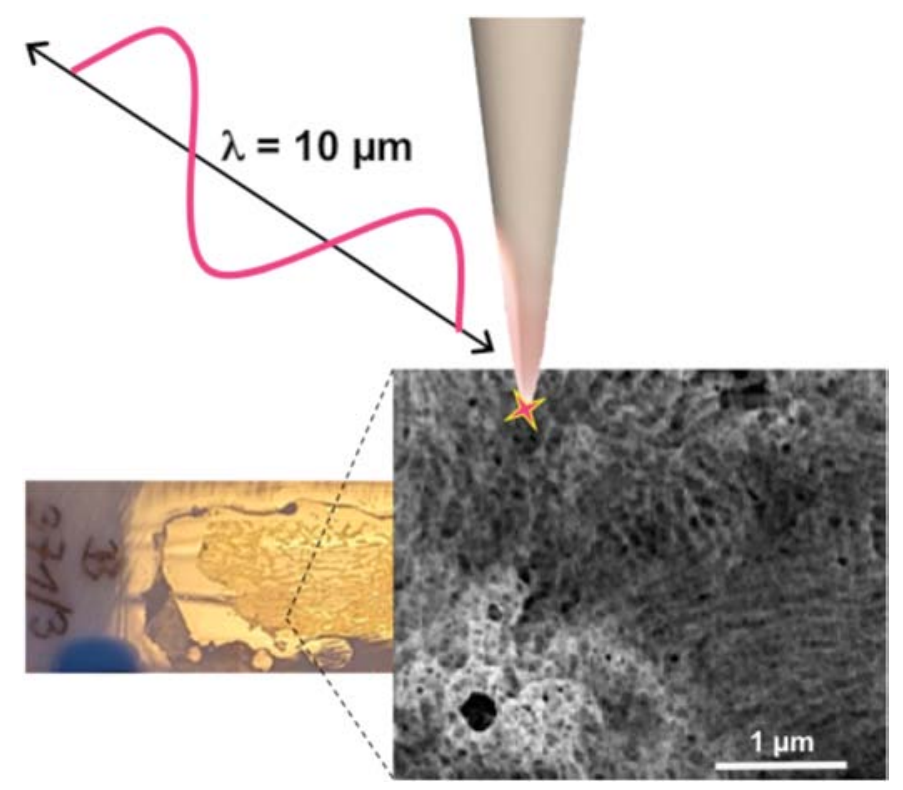

Figure 3: IR nanoscopy of polished human bone sample. S-SNOM probing is sketched here, with the probing wavelength of $9.47 \mu \mathrm{m}$ and the tip at approximately the same scale as the IR image of apatite nanocrystals surrounding a canaliculus [4].

and requires conductive coatings, SEM images were taken only after completion of s-SNOM imaging because s-SNOM is sensitive to damage as it probes a thin surface layer only (of 20-50 $\mathrm{nm}$ depth) [2].

Organic materials for solar cells. Solar cells converting sunlight directly into electric power are installed all over the world; these mostly consist of single-crystal Si material to optimize photon-induced charge production and transport. The minimization of structural defects that could scatter charge carriers allows a conversion efficiency that is currently about $25 \%$ [7]. The quest for lower-cost alternative materials is proceeding with great international effort. Organic solar cells consist of suitable conductive polymers or small organic molecules that facilitate large-area flexible devices and low-cost production on many types of substrate by wet-chemical processing or thermal evaporation. In the case of well-defined small molecules, the resulting organic thin films are highly ordered, but they are far from being defectfree. They show a crystalline grain structure on a length-scale on the order of $1 \mu \mathrm{m}$ (Figure 4a). Key challenges for organic solar cell development are these materials' short diffusion length, due to a high density of structural defects and a short carrier scattering time. Certainly the nanoscale morphology is a key issue for charge-transport properties, and accordingly, great effort has been made toward morphology control [8]. It achieved device conversion efficiency up to $11 \%$ [7]. However, it is very difficult to map the nanoscale crystallization, and it remains unclear where the electronic defects are localized.

Nanoscale polymorphism in thin-film pentacene. Today's best-performing organic solar cells consist of thin films with optimally designed, synthesized molecules. Pentacene has long been the prototypical molecule to study the fundamental electronic and structural properties of organic thin films. It is a small molecule made up as a linear chain a Topography
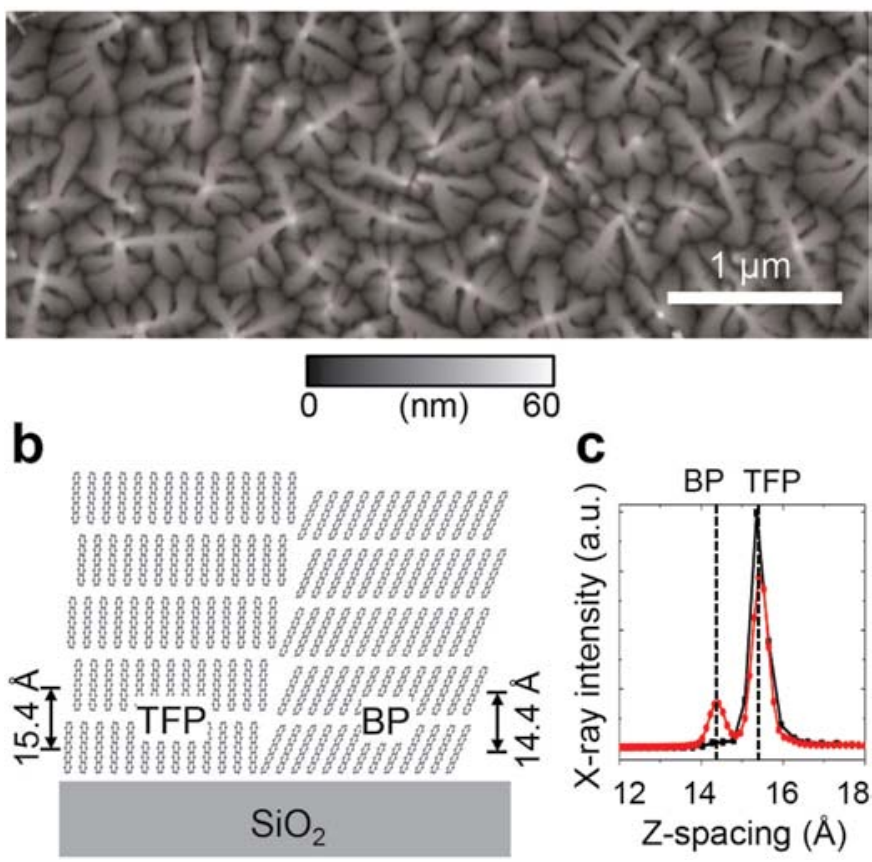

Figure 4: Pentacene film (about $40 \mathrm{~nm}$ thick) on $\mathrm{Si}$, after 20 months of storage. (a) Topography showing a grain structure. (b) Sketch of molecular order for two adjoining polymorphs, thin-film phase (TFP) and bulk phase (BP). (c) Result of $\mathrm{X}$-ray scattering (linear scale) to determine average molecular layer period after preparation (black) and stored for 20 months (red) [5].

of five benzene rings. On evaporation, pentacene molecules stand almost perpendicular to the $\mathrm{SiO}_{2}$ substrate surface. Continuous thin (typically $40 \mathrm{~nm}$ ) pentacene films of ordered crystalline grains (Figure $4 \mathrm{~b}$ ) can be p-type semiconductors with mobility up to $10 \mathrm{~cm}^{2} / \mathrm{Vs}$, comparable to amorphous Si. X-ray diffraction aimed at determining the molecular layer period shows two distinctly differing values (Figure 4c). This means that two distinct polymorphs, the thin-film phase (TFP) and the bulk phase (BP), coexist and even change over time. The footprint of the X-ray beam on the sample is macroscopic, of the order of $1 \mathrm{~mm}^{2}$, and until recently no available microscopy could decide the important question of how both polymorphs may be spatially distributed, and over which lateral or vertical dimensions (see Figure $4 \mathrm{~b}$ for a possible arrangement). Infrared nanoscopy now straightforwardly decides these issues [5].

The essential reason for this success is that the two structural phases differ in their vibration frequencies. For Figure $5 b$ the IR frequency was chosen to highlight the BP polymorph. Indeed there are elongated BP ellipsoidal domains of about $400 \mu \mathrm{m}$ in width everywhere on the sample, obviously embedded in TFP pentacene. They do not appear to be correlated to the topographic morphology because they even extend through grain boundaries. A repeat of the image acquisition with the illumination frequency changed to highlight TFP pentacene produces an IR image with reversed contrast, as would be expected. As a final experimental proof, nano-FTIR spectra were acquired at different positions of the sample (Figure $5 \mathrm{c}$ ). Evidently the local spectrum taken on a BP ellipsoid shows a higher resonance frequency $\left(906 \mathrm{~cm}^{-1}\right)$ than that of the surrounding TFP $\left(904 \mathrm{~cm}^{-1}\right)$. These values are consistent with published far-field FTIR-spectra of the two polymorphs. Note that 

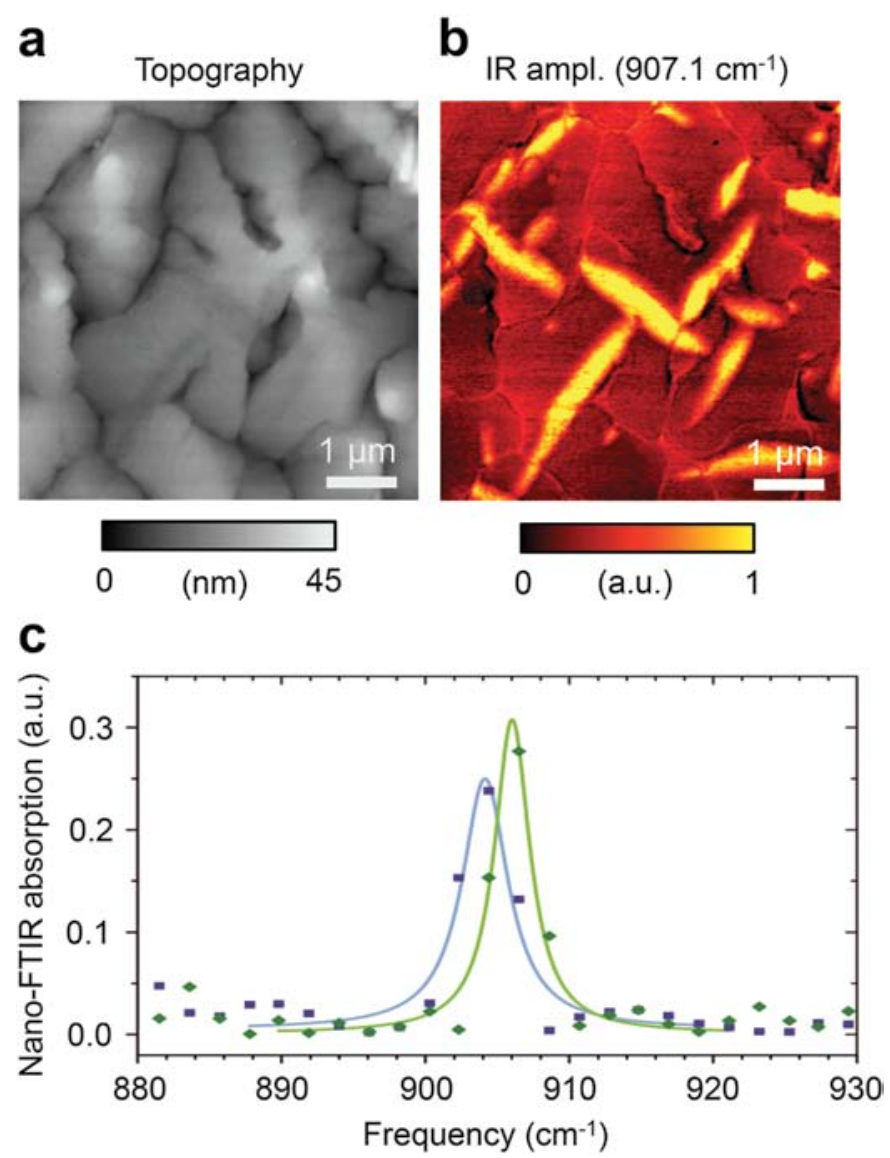

Figure 5: High-resolution IR nanoscopy of a similar pentacene film as in Figure 4a. (a) Topography, (b) simultaneously recorded IR map at $907.1 \mathrm{~cm}^{-1}$ (corresponding to $11 \mu \mathrm{m}$ wavelength, resonant with BP pentacene), and (c) nano-FTIR spectra at two locations, on and off a BP ellipsoid domain [5].

a basic agreement of far-field and near-field resonance positions of molecules has been recently confirmed in experiment and theory [6]. Lastly, it may be questioned whether the polymorphs extend throughout the film depth, as sketched in Figure 4b, or whether there may be a sign of inhomogeneity also in the vertical direction. The answer is negative from the following observation: the topography (Figure 5a) shows on close inspection a depression of about $2-3 \mathrm{~nm}$ at all BP elliptic regions. Given the film thickness of $40 \mathrm{~nm}$, the depression amounts to about $6 \%$ shrinkage, and this agrees quantitatively with the lowering of vertical extent of a BP $(1.44 \mathrm{~nm}=14.4 \AA)$ compared to a TFP pentacene molecule $(1.54 \mathrm{~nm}=15.4 \AA)$. Thus an inhomogeneous ordering of TFP and BP phases in the vertical direction can be ruled out. In fact it is highly probable that an initially homogeneous TFP pentacene film over time changes its conformation, by nucleation and lateral growth of vertically homogeneous BP polymorph ellipsoid domains, keeping the number of molecular layers unchanged [5].

\section{Discussion}

A straightforward, independent proof of our findings could come from X-ray microscopy at brilliant light sources like synchrotrons, which promise comparable resolution in the near future. Quite generally, it will be interesting and exciting to see IR nanoscopy results confirmed and strengthened by complementary high-resolution techniques, as already performed with SEM $[3,4]$.

\section{Conclusion}

Infrared nanoscopy, performed in the IR near-field microscope, is able to produce either local IR spectra or images using selected wavelengths at spatial resolutions down to $20 \mathrm{~nm}$. This allows routine identification of tiny crystals embedded in various matrices.

\section{Acknowledgement}

We are grateful to S. Amarie, C. Westermeier, P. Zaslansky, Y. Kajihara, E. Griesshaber, W.W. Schmahl, T. Geith, S. Milz, F. Bamberg, A. Cernescu, and B. Nickel for their original contributions and active collaboration in developing IR nanoscopic applications.

\section{References}

[1] L Novotny and B Hecht, Principles of Nano-Optics, Cambridge University Press, New York, 2007.

[2] F Keilmann and R Hillenbrand, "Near-field nanoscopy by elastic light scattering from a tip" in Nano-Optics and Near-Field Optical Microscopy, eds. A Zayats and D Richards, Artech House, Norwood, MA, 2009.

[3] S Amarie et al., Beilstein J Nanotech 3 (2012) 11.

[4] T Geith et al., J Biophotonics (7) (2014) 418-24.

[5] C Westermeier et al., Nat Commun 5 (2014), article 4101, doi:4110.1038ncomms5101.

[6] F Huth et al., Nano Lett 12 (2012) 3973-78.

[7] N.R.E. Laboratory http://www.nrel.gov/ncpv/images/ efficiency_chart.jpg, accessed Sept. 2014.

[8] Y Lin et al., Chem Soc Rev 41 (2012) 4245-72.

\section{Amptek FAST SDD ${ }^{\circledR}$ for EDS (SEM) Applications}

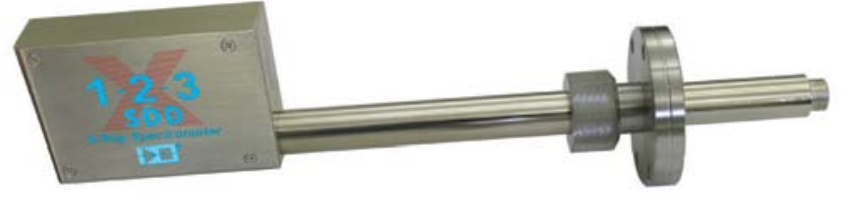

Amptek's FAST SDD ${ }^{\circledR}$ detector for EDS use with SEMs utilizes new technology "C2 Series" X-ray windows (Si3N4) and has a low energy response down to Boron. With its high intrinsic efficiency the SDD is ideal for EDS XRF. See why Amptek detectors are the \#1 choice of OEMs worldwide.

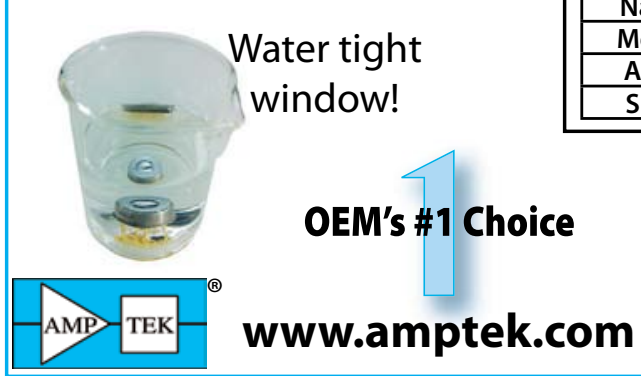




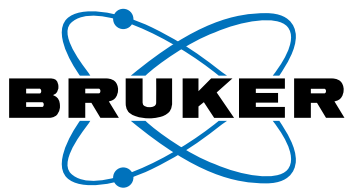

\section{5-in-1 fantasy: Giralope.}

\section{5-on-1 reality: Bruker's range of analytical tools for SEM.}

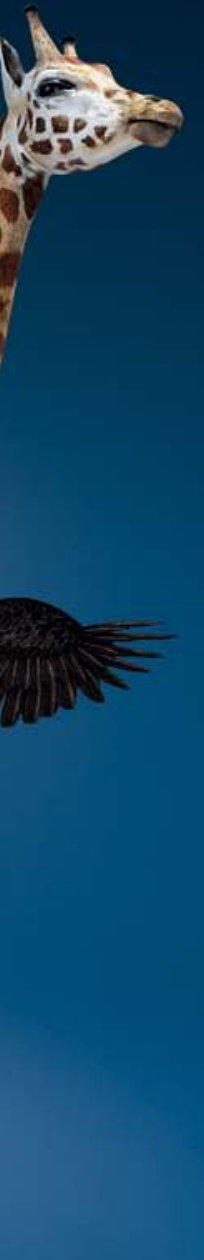

